\title{
CORRECTION
}

Erdal Öner • Ahmet Birinci

\section{Correction to: Investigation of the solution for discontinuous contact problem between a functionally graded (FG) layer and homogeneous half-space}

Published online: 7 October 2020

(c) Springer-Verlag GmbH Germany, part of Springer Nature 2020

\author{
Correction to: Arch Appl Mech \\ https://doi.org/10.1007/s00419-020-01750-y
}

Unfortunately, Figs. 5, 6, 8 and 10 have been processed incorrectly. Correct figures are provided in this correction article.

The Original article has also been corrected.

The original article can be found online at https://doi.org/10.1007/s00419-020-01750-y

E. Öner $(\varangle)$

Department of Civil Engineering, Bayburt University, 69000 Bayburt, Turkey

E-mail: eoner@bayburt.edu.tr

A. Birinci

Department of Civil Engineering, Karadeniz Technical University, 61080 Trabzon, Turkey

E-mail: birinci@ktu.edu.tr 


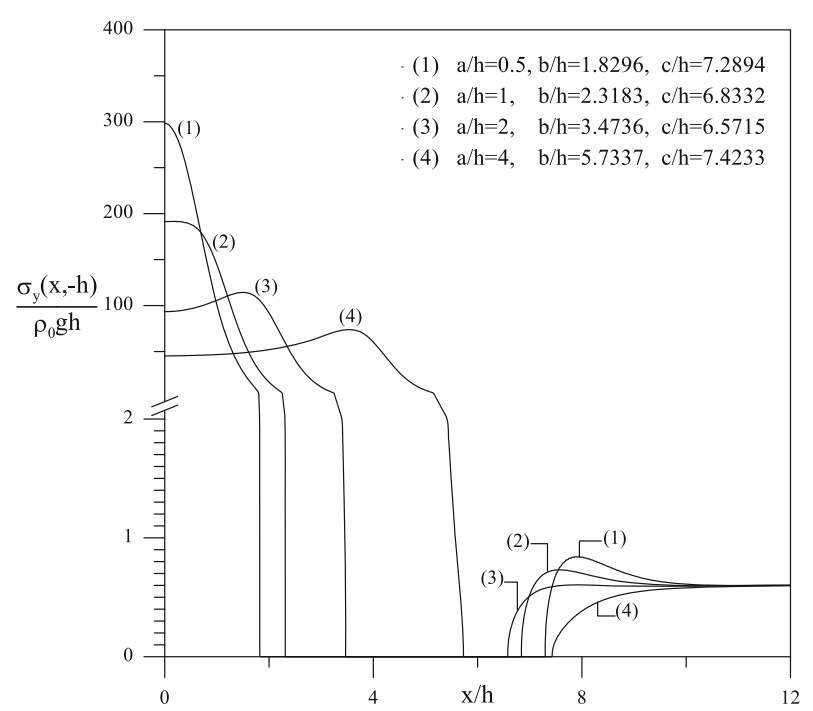

Fig. 5 The changes in the dimensionless contact stress distributions between FG layer and homogeneous half-space depending on block width $(\mathrm{a} / \mathrm{h})\left(\kappa_{1}=\kappa_{2}=2, \beta \mathrm{h}=-0.6931, \gamma \mathrm{h}=1.0986, \lambda=500>\lambda_{\mathrm{cr}}, \mu_{-\mathrm{h}} / \mu_{2}=1\right)$

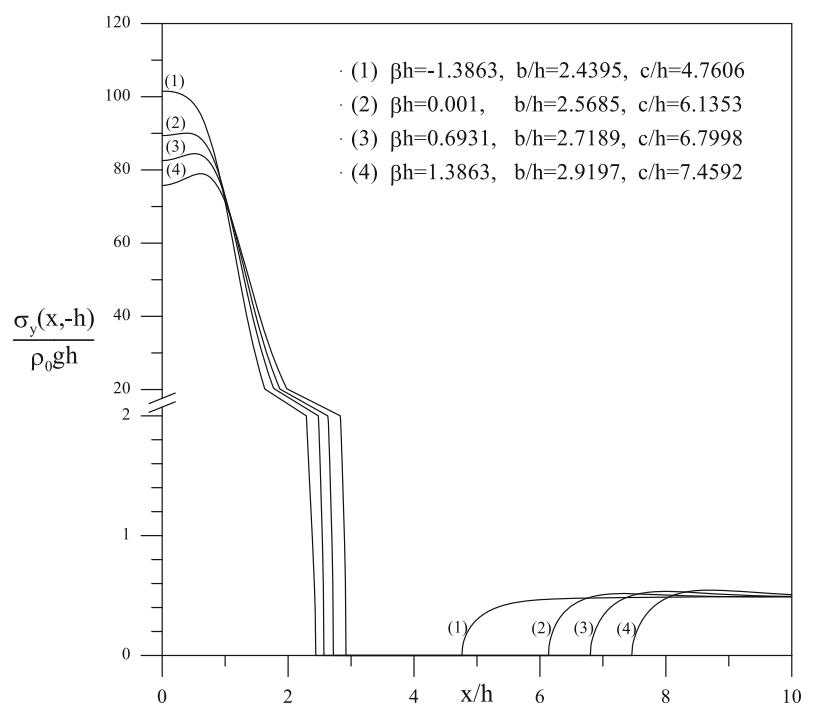

Fig. 6 The changes in the dimensionless contact stress distributions between FG layer and homogeneous half-space depending on rigidity parameter $(\beta \mathrm{h})\left(\kappa_{1}=\kappa_{2}=2, \mathrm{a} / \mathrm{h}=1, \gamma \mathrm{h}=1.6094, \lambda=250>\lambda_{\mathrm{cr}}, \mu_{-\mathrm{h}} / \mu_{2}=1\right)$ 


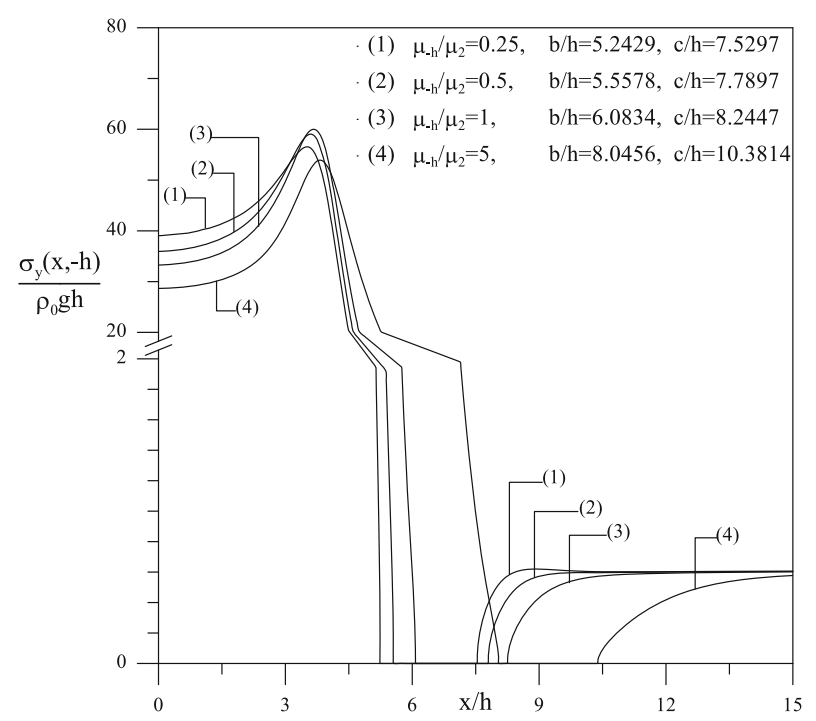

Fig. 8 The distributions of the dimensionless contact stresses $\sigma_{\mathrm{y}}(\mathrm{x},-\mathrm{h}) /\left(\rho_{0} \mathrm{gh}\right)$ between FG layer and homogeneous half-space depending on $\mu_{-\mathrm{h}} / \mu_{2}$ ratio $\left(\kappa_{1}=\kappa_{2}=2, \mathrm{a} / \mathrm{h}=4, \beta \mathrm{h}=0.6931, \gamma \mathrm{h}=1.0986, \lambda=400>\lambda_{\mathrm{cr}}\right)$

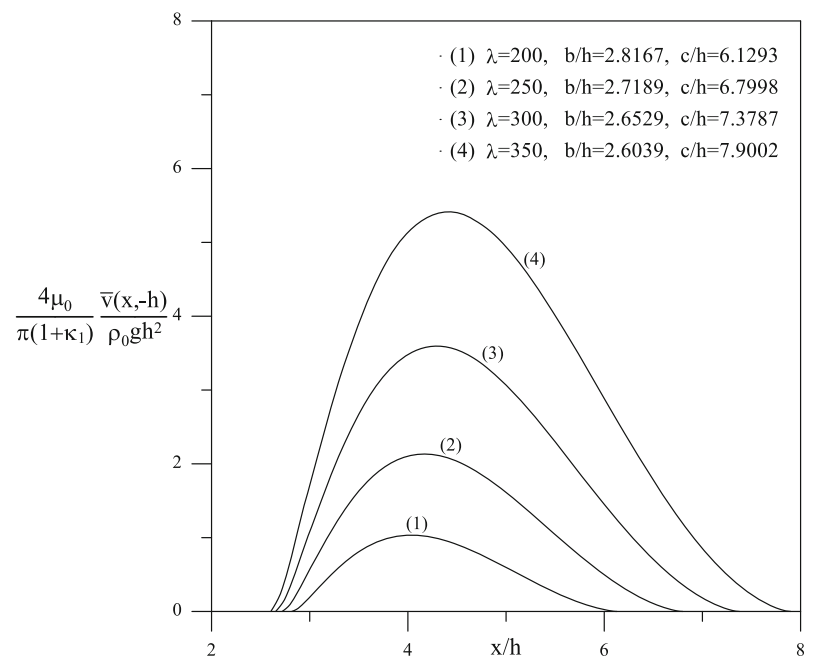

Fig. 10 Variation of separation displacement $\bar{v}(\mathrm{x},-\mathrm{h})$ between FG layer and homogeneous half-space depending on load factor $(\lambda)\left(\kappa_{1}=\kappa_{2}=2, \mathrm{a} / \mathrm{h}=1, \beta \mathrm{h}=0.6931, \gamma \mathrm{h}=1.6094, \mu_{-\mathrm{h}} / \mu_{2}=1\right)$

Publisher's Note Springer Nature remains neutral with regard to jurisdictional claims in published maps and institutional affiliations. 